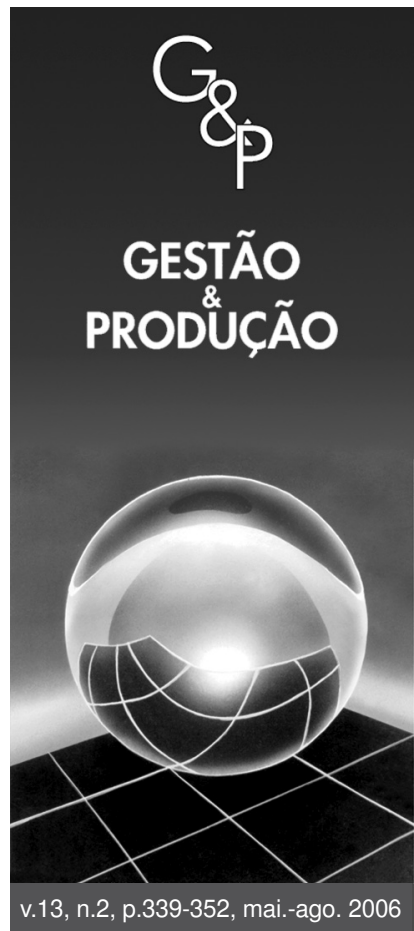

\title{
AVALIAÇÃO MULTICRITERIAL DE DESEMPENHO: UM ESTUDO DE CASO NA INDÚSTRIA DE TRANSPORTE COLETIVO DE PASSAGEIROS
}

\author{
Miguel Afonso Sellitto \\ Miriam Borchardt \\ Giancarlo Medeiros Pereira
}

Programa de Pós-Graduação em Engenharia de Produção e Sistemas, Universidade do Vale do Rio dos Sinos - Unisinos, Av. Unisinos 950, 93022-000, São Leopoldo, RS, e-mails: sellitto@unisinos.br, miriamb@unisinos.br, gian@unisinos.br

Resumo

O objetivo deste trabalho é apresentar uma proposta de estrutura multicriterial de avaliação de desempenho em organizações. $O$ trabalho inicia descrevendo e contextualizando conceitos relativos aos sistemas de medição de desempenho em organizações. Também descreve modelos de gestão baseados nos critérios de excelência do prêmio Malcolm Baldridge e na teoria da mentalidade enxuta. A seguir propõe um método para a construção de uma estrutura flexível de avaliação de desempenho e descreve um caso de aplicação que a exemplifica: a avaliação do desempenho de aspectos da mentalidade enxuta inseridos em uma operação de transporte coletivo. Ao final discutem-se os resultados do caso, comparam-se estes com o método de avaliação vigente em duas empresas da indústria e encaminha-se a continuidade da pesquisa.

Palavras-chave: avaliação multicriterial de desempenho, avaliação de desempenho em produção enxuta, avaliação de desempenho em transporte coletivo.

\section{Introdução}

Uma parte relevante do planejamento estratégico de organizações é a medição e o controle dos resultados da aplicação das estratégias de operação. Se a medição for desconectada dos objetivos estabelecidos no processo estratégico, estes podem não ser atingidos pelos planos de ação. Se os resultados destes planos não se subordinarem aos objetivos estratégicos, por uma estrutura de medição e controle, os esforços podem resultar contraditórios e ineficazes.

Para Bititci et al. (2001) e Kennerly e Neely (2003), a natureza dos ambientes competitivos atuais tem exigido mais responsividade das empresas. A gestão de fatores, tais como a qualidade, a velocidade nas entregas, a confiabilidade nos prazos e a flexibilidade em serviços também têm sido apontadas como importantes na obtenção dos objetivos financeiros. Tais fatores sempre foram relevantes para o resultado empresarial, porém, em um ambiente mais previsível, é mais fácil corrigir desvios nestes fatores a partir de quedas nos resultados financeiros finais. Os autores concluem que novos métodos de medição são oportunos, incluindo medições multidimensionais e variáveis intermediárias, as variáveis de estado, cujo desempenho antecipe a tendência dos resultados finais das estratégias em andamento.

Bititci et al. (2002), Gomes et al. (2004) e Melnick et al. (2004) apresentaram resenhas parciais de estruturas e metodologias já propostas, das quais se apontam algumas e suas datas de publicação: (i) o modelo do prêmio Malcolm Baldridge, em 1987; (ii) o modelo SMART, em 1989; (ii) o questionário para a medição de desempenho, em 1990; (iv) o BSC, em 1992 e 1996; (v) o PMSDP, 
pelo grupo de pesquisa de Cambridge, em 1996; (vi) o modelo de referência IPMS, de 1998; (viii) o modelo de impacto financeiro de desempenho; e (ix) o Performance Prism, de 2001. Miranda e Silva (2002) apontam vinte e um modelos publicados após 1990, todos propondo o uso de medidas não-financeiras. Segundo estes autores, muitas das metodologias são estáticas e históricas, não percebendo mudanças nos ambientes externos e internos e tornando irrelevante a informação disponível.

A partir de agora, fala-se em avaliação de desempenho, quando esta se der por julgamentos de variáveis categóricas, e em medição de desempenho, quando esta se der por medições de variáveis quantitativas. Princípios similares podem ser usados na escolha de variáveis a serem avaliadas e a serem medidas.

O objetivo geral deste trabalho é propor uma metodologia de construção de uma estrutura flexível multicriterial de avaliação de desempenho. A estrutura deve ser capaz de capturar os condicionantes da execução de uma estratégia e ser facilmente modificada sempre que variem estes condicionantes. A proposta será testada e exemplificada em um caso específico: a avaliação do desempenho de aspectos extraídos da teoria da mentalidade enxuta e inseridos em uma operação de transporte coletivo.

Os objetivos secundários são: (i) aplicar a metodologia em um caso de operação de transporte coletivo; (ii) comparar a aplicação com uma estrutura usada na indústria estudada, baseada nos critérios de excelência do prêmio Malcolm Baldridge; e (iii) discutir e refinar o modelo para outras aplicações. Espera-se que o estudo de caso incorpore peculiaridades que escaparam à proposição, refine e torne o método mais robusto para outras aplicações.

Os multicritérios de avaliação serão ponderados pelo método AHP (Analytic Hierarchy Process), pelo modo com que o método trata e constrói a estrutura de avaliação, o que é corroborado por Liu e Hi (2005), que afirmam que "a mais forte característica do AHP é gerar prioridades numéricas a partir do conhecimento subjetivo expresso em comparações pareadas matriciais". O método é apresentado em Saaty (1991), é abundantemente explorado na literatura (Partovi et al., 2002; Liu e Hai, 2005) e, para Salomon e Montevechi, apud Salomon (2002), é, possivelmente, o método multicriterial mais conhecido e mais usado. Dyer (1990a; 1990b) apresenta críticas ao método, detendo-se principalmente nas alterações que surgem na ordenação se alternativas são adicionadas. Réplicas surgem em Saaty (1990) e Harker e Vargas (1990). Sellitto (2005) aponta e discute o mesmo fenômeno quando um critério de decisão é retirado. Gomes et al. (2004) e Liu e Hai (2005) apresentam alternativas ao método. Mello et al. (2002) comparam o AHP ao MACBETH e afirmam que este é consistente, enquanto aquele admite inconsistência de até $10 \%$. Ob- serva-se que esta inconsistência é arbitrária e um nível menor pode ser admitido.

A contribuição essencial do artigo é a formulação multicriterial para o problema, destacando-se que os resultados específicos do caso podem ter inconsistências devidas às deficiências do AHP, que não serão discutidas. Outros métodos são remetidos à continuidade.

Há interesse de pesquisa pelo tema (a literatura fala mais em medição do que avaliação de desempenho). Cem dos 2.651 artigos apresentados nos ENEGEP de 2001 a 2004 citam a expressão medição de desempenho. Cinco teses recentes trataram da medição de desempenho em organizações (Martins, 1999; Müller, 2003; Gasparetto, 2003; Hansen, 2004 e Sellitto, 2005) e uma tese recente tratou da mentalidade enxuta na indústria do transporte coletivo (Borchardt, 2005); todas usadas na pesquisa. Lima (2000) e Lima (1999) trataram de avaliação multicriterial de desempenho no transporte coletivo. Sellitto (2005) resume o interesse atual de pesquisa internacional no tema.

O artigo se estrutura em: (i) revisão sobre medição de desempenho, mentalidade enxuta e modelos de avaliação baseados nos critérios de excelência do prêmio Malcolm Baldridge; (ii) metodologia e caso; (iii) discussão; e (iv) conclusões e continuidade. O artigo apresenta os resultados do segundo ano de um projeto de pesquisa de três anos sobre operações industriais, conduzido pela Engenharia de Produção da UNISINOS.

\section{Medições de desempenho em organizações}

O interesse pela medição de desempenho cresceu a partir dos anos 1990. Segundo Martins (1999), os sistemas de medição mais encontrados até então se preocupavam mais com resultados financeiros do que com outras dimensões de desempenho. Metas exclusivamente financeiras, tais como o lucro líquido e a taxa de retorno, eram referencial de desempenho. Segundo Bourne et al. (2000) e Kennerly e Neely (2003), tais medições acarretam, entre outros problemas: (i) falta de informação sobre o desempenho futuro; (ii) enfoque em otimizações de curto prazo e locais; (iii) perda de alinhamento com estratégias mais elaboradas de produção; e (iv) pouca informação para decisões em ambientes turbulentos.

Segundo Kaplan e Norton (1997), medições exclusivamente financeiras não são eficazes no controle estratégico de uma organização produtiva. Segundo Suwignjo et al. (2000), pesquisas recentes comprovaram que medições financeiras não são capazes de medir e integrar todos os fatores críticos exigidos para o sucesso de uma operação. Miranda e Silva (2002) concluem que medidas financeiras são suficientes apenas se a concorrência não é acirrada, as mudanças são lentas ou há poucas modi- 
ficações nos produtos. Hansen (2004) sugere a inclusão de valores intangíveis, tais como o capital intelectual e o aprendizado, em estruturas hierarquizadas de medição de operações complexas.

Segundo Sellitto (2005), dois interesses distintos são atendidos por medições de desempenho: (i) o público externo - tal como o mercado acionário, o poder público e os sindicatos -, atendido por balanços patrimoniais e sociais; e (ii) o público gerencial interno, atendido por sistemas de informação estruturados para a medição ou avaliação de desempenho.

Para Miranda e Silva (2002), a principal função da medição de desempenho é verificar se e em que grau a missão da organização está sendo cumprida. Segundo os autores, ao estruturar-se um sistema de medição de desempenho, três perguntas devem ser respondidas: (i) por que medir, exigindo clareza acerca da missão; (ii) o que medir, exigindo que os principais campos de força sejam explicitados e entendidos; e (iii) como medir, exigindo um processo de relacionamento entre as grandezas que operam os campos de força, geralmente latentes, e variáveis manifestas que possam capturar e descrever o seu comportamento.

Para Melnick et al. (2004), a medição de desempenho é o elemento que conecta estratégia e realidade: estratégia sem medição é inútil, medição sem estratégia não faz sentido. Para Hronec (1993), as medições de desempenho são os sinais vitais da organização, comunicando a estratégia para baixo e os resultados para cima e englobando a totalidade do entendimento acerca dos objetivos e das relações subjacentes de causa-efeito. Para Moreira (1996), um sistema de medição de desempenho organizacional é um conjunto de medidas referentes à organização como um todo e às suas partições, processos e atividades. Para Müller (2003), o monitoramento por medições de desempenho torna possível a melhoria e a comparação dos processos com as melhores práticas, o benchmarking. Para Bourne et al. (2000), a medição de desempenho tem duas funções: medir o sucesso de uma estratégia e verificar a validade dos pressupostos assumidos ao se formular a estratégia. Para Gasparetto (2003), os sistemas de avaliação de desempenho devem considerar os aspectos sistêmicos da organização, integrando indicadores segundo uma regra vinculada à estratégia e devem ser alterados se a estratégia ou o cenário de competição modificar-se. Para Moreira (1996), as medições devem estar alinhadas com a visão de futuro e com as estratégias atuais, partindo da visão e descrevendo as estratégias. Para Arantes (1998) e Tonchia (2000), o desempenho deve ser medido por resultados finais, que justifiquem a existência da organização, e por resultados intermediários, que expressem o modo pelo qual se acredita que os resultados serão obtidos.
Para Kasul e Motwani (1995), antes de se propor um sistema de medição de desempenho, deve-se conhecer os fatores críticos para o sucesso da estratégia. Os autores apontaram nove classes de fatores críticos que devem ser medidos em uma operação de classe mundial: (i) compromisso gerencial; (ii) qualidade; (iii) serviços ao cliente; (iv) fornecimentos; (v) tecnologia; (vi) instalações; (vii) flexibilidade nas operações; (viii) preços e custos; e (ix) competitividade global. Os autores descreveram cinqüienta e oito medidores que podem suportar os nove requisitos mencionados.

Bourne et al. (2000) dividem o processo de implantação da medição de desempenho em especificação, implementação e uso das medições. Segundo os autores, a especificação das medições é um processo cognitivo em que objetivos de estrategistas são transformados em medições capazes de mensurá-los. A implementação é gerenciável por metodologias de controle de projeto e baseada em tecnologia de informação. Por fim, a fase de uso consiste na atualização e manutenção das medições, em um processo permanente de aprendizado. Lohman et al. (2004) também apontam etapas para a construção de um sistema de medição de desempenho, destacando como última etapa o questionamento da validade dos indicadores utilizados e os testes para a eventual atualização.

Kennerly e Neely (2003) observam que as empresas têm atribuído menos atenção à fase de atualização das medições. Segundo os autores, há pouca evidência de que, havendo mudanças no ambiente de negócios ou na estratégia, o sistema de medição continue refletindo as necessidades organizacionais. Para Bourne et al. (2000), o sistema de medição de desempenho deve prever um procedimento de inserção, remoção e revisão de medições, executável sempre que sejam detectadas modificações no ambiente ou na estratégia de competição adotada.

\subsection{Multicriterialidade e hierarquias nas medições de desempenho}

Melnick et al. (2004) falam em medições individuais, grupos de medições individuais e medições agregadas, das quais se obtém um senso de desempenho global para o grupo de medições, segundo as premissas de agregação. Sellitto (2005) aponta que, em um sistema de medição de desempenho, as medições são os elementos e as hierarquias e composições são as relações. As medições podem ser agrupadas segundo afinidades e resumidas em medições intermediárias mais gerais, em um formato arborescente.

As relações no sistema de medições não se limitam às hierarquias estruturais. Para Bititci (1995), um sistema de medição de desempenho deve prever interações entre seus elementos, capazes de: (i) evitar a subotimização local; (ii) desdobrar os objetivos estratégicos até os níveis operacionais; (iii) proporcionar o pleno entendimento da estrutura 
de objetivos e de conflitos, os trade-offs da estratégia; e (iv) considerar aspectos da cultura organizacional.

O uso de múltiplas variáveis na medição de desempenho remete à decisão multicriterial. Para French (1986), dificilmente em uma decisão multicriterial se tem um modelo clara e unicamente estruturado. $\mathrm{O}$ decisor deve perceber que atributos podem interferir no objetivo, formando uma primeira camada de critérios e, para cada critério, repetir o desdobramento, até que todos os fatores importantes tenham sido relacionados em uma estrutura arborescente. Atribuídos pesos aos critérios, por alguma técnica multicriterial de apoio à decisão, a comparação entre duas empresas avaliadas pelas mesmas $n$ variáveis de estado se torna um problema de decisão multicriterial de comparação entre dois vetores $n$-dimensionais.

Discussões mais aprofundadas sobre a teoria da decisão com enfoque multicriterial são encontradas em Keeney e Raiffa (1976) e em French (1986).

Um sistema de medições de desempenho assume um caráter hierárquico ao usar variáveis de estado intermediárias para representar a realidade complexa de uma estratégia ou operação. Uma hierarquia é construída a partir do conhecimento do decisor acerca da situação de interesse. Caso já haja um estudo teórico, é possível construir a hierarquia a partir da própria teoria, pois esta, geralmente, separa e classifica o conhecimento acerca do objeto em estudo.

Uma hierarquia é uma abstração da estrutura de uma complexidade, cujo objetivo é explicar as interações intermediárias e as intensidades de seus impactos na função global. Em uma hierarquia, um ente superior é influenciado por entes inferiores, que por sua vez são influenciados por outros entes sucessivamente inferiores, em uma estrutura descendente. Um grupo de entes de mesmo nível influencia apenas o grupo imediatamente superior e é influenciado apenas pelo grupo imediatamente inferior, não havendo subordinação múltipla. Os critérios inferiores devem ser mutuamente exclusivos e fornecer uma descrição exaustiva do critério superior. Para cada critério superior, deve haver, ao menos, dois critérios inferiores para que se justifique a estrutura arborescente (Ensslin, et al., 2001; Keeney apud Ensslin et al., 2001; Saaty, 1991).

Para este trabalho interessam as hierarquias de níveis mutuamente independentes e lineares, sem relações circulares. Outras hierarquias são discutidas em Salomon e Montevechi (1998).

\section{Estruturas de desempenho baseadas nos critérios de excelência do prêmio Malcolm Baldridge}

Evans (2004) destaca o prêmio Malcolm Baldridge como uma alternativa relevante para a geração de modelos de medição de desempenho empresarial. Na indústria do transporte coletivo da capital estudada, esta alternativa é difundida em vários formatos (PNQ, ANTP, PGQP) e será utilizada na pesquisa, portanto entende-se que seja útil revisá-la.

Sellitto (2005) classifica os modelos baseados no prêmio Malcolm Baldridge como metodologias fortemente estruturadas, pois apresentam hierarquias e ponderações fixas. As estruturas destes modelos variam apenas em intervalos de tempo longos e após análises e consensos institucionais, não se podendo dizer que sejam flexíveis, pois as modificações, quando acontecem, são muito mais lentas do que as modificações nos ambientes estratégicos. Em contrapartida, estes modelos formam séries históricas próprias para comparações.

Os modelos derivam de um modelo original, o Malcolm Baldrige National Quality Award, criado em 1987, com o objetivo de promover a busca da excelência em organizações públicas e privadas, de negócios, saúde e educação. Entre os objetivos específicos do prêmio, há um método padronizado de avaliação de desempenho, que mensura o termo teórico excelência na gestão, suportado por sete construtos apoiados em dezenove conceitos, representados por variáveis categóricas. A mensuração global é linear e em arborescência (BNQP, 2003).

Entidades brasileiras propõem prêmios similares, com modificações em variáveis e pesos, tais como: a Fundação Nacional para a Qualidade, a FNQ, antiga FPNQ, que administra o Prêmio Nacional da Qualidade, o PNQ; a Associação Nacional de Transportadores de Passageiros, a ANTP; o Programa Gaúcho de Qualidade e Produtividade, o PGQP; e a ABES, Associação Brasileira de Engenharia Sanitária. Internacionalmente, entre outros, destacam-se os prêmios de qualidade europeus e iberoamericano e o prêmio Shingo de excelência na manufatura.

Dentre os modelos usados na indústria estudada, será revisado o PNQ. O modelo assume como premissa a adoção do TQC, estruturando o termo teórico excelência empresarial em oito critérios: liderança, estratégias e planos, clientes, sociedade, informações e conhecimento, pessoas, processos e resultados. Os critérios são construtos latentes suportados por vinte e sete conceitos, cuja avaliação se dá por julgamento de variáveis categóricas descritoras das definições subjacentes ao conceito (FPNQ, 2004).

Segundo a FPNQ (2004), a sobrevivência e o sucesso de uma organização estão relacionados à capacidade de atender às necessidades de clientes, que devem ser identificadas, entendidas e utilizadas na gestão de produtos e serviços. Para que haja continuidade, deve-se identificar, entender e satisfazer as necessidades da sociedade e das comunidades, cumprindo as leis e preservando os ecossistemas. A liderança estabelece os valores e as diretrizes 
da organização, pratica e vivencia as bases da excelência e impulsiona, pelo exemplo, sua disseminação na organização. Os líderes são os responsáveis por resultados que assegurem a satisfação das partes interessadas, analisando criticamente o desempenho e tomando ações de controle e aprendizado organizacional. As estratégias de competição são desdobradas em planos de ação. Para comunicar as estratégias e mensurar o desempenho, é planejado e operado um sistema de informação e conhecimento, cujos resultados realimentam as ações de controle e, pelo aprendizado, formam a inteligência e a cultura da excelência da organização.

Os critérios de avaliação do PNQ e seus pesos, como usados na pesquisa, são apresentados na Tabela 1.

\section{A mentalidade enxuta em operações}

A mentalidade enxuta é um conjunto de práticas de gerenciamento que nasceu da dificuldade das empresas de fabricação em manter a lógica da produção em massa a partir dos anos 1990. Esta lógica gerou distorções, tais como longos tempos para o lançamento de novos produtos e ineficiência em cadeias logísticas, causadas, principalmente, por foco gerencial voltado a requisitos de qualidade e desempenho, em detrimento a requisitos de competição. Quando os requisitos dos clientes passaram a incluir a diferenciação no produto e a rapidez de entrega, as empresas mudaram as estratégias, passando a considerar a experiência japonesa e técnicas, tais como a reengenharia e a análise de valor, que foram codificadas na forma da mentalidade enxuta e resumidas em cinco princípios (Womack e Jones, 1997):

- Especificação do valor a partir da perspectiva do cliente final. O valor do bem ou serviço é especificado para atender à necessidade específica do cliente, em um prazo satisfatório e por preço e quantidade adequados, evitando-se a redução de custos, a aplicação do "estadoda-arte" ou o privilégio aos trabalhadores como objetivo das ações;

- Identificação e mapeamento da cadeia geradora do valor. O mapeamento das ações de produção de um bem ou serviço permite que se conheça o estado atual e as perdas na cadeia e, logicamente, discernir as ações para sua eliminação. Womack e Jones (1997) caracterizam três casos: ações que criam valor percebido pelo cliente; ações que não criam valor, mas das quais não se pode abrir mão; e ações que não criam valor e podem ser eliminadas.

- Aceleração do fluxo nas cadeias. O que sobrou na cadeia deve fluir o mais livre possível, reduzindo os tempos de atravessamento nas atividades;

- Estabelecer um sistema de produção puxada pelo cliente. Após acelerar o fluxo, deve-se equilibrá-lo com a necessidade do cliente, que determina os ritmos, prazos
Tabela 1. Critérios de pontuação do PNQ (Fonte: FPNQ, 2004).

\begin{tabular}{|c|c|}
\hline Critérios e itens de avaliação & $\begin{array}{c}\text { Pontuação } \\
\text { máxima }\end{array}$ \\
\hline 1 - Liderança & 100 \\
\hline 1.1 - Sistema de liderança & 30 \\
\hline 1.2 - Cultura da excelência & 40 \\
\hline 1.3 - Análise crítica do desempenho global & 30 \\
\hline 2 - Estratégias e Planos & 90 \\
\hline 2.1 - Formulação das estratégias & 30 \\
\hline 2.2 - Desdobramento das estratégias & 30 \\
\hline $\begin{array}{l}2.3 \text { - Planejamento da medição do desempe- } \\
\text { nho }\end{array}$ & 30 \\
\hline 3 - Clientes & 60 \\
\hline 3.1 - Imagem e conhecimento do mercado & 30 \\
\hline 3.2 - Relacionamento com clientes & 30 \\
\hline 4 - Sociedade & 60 \\
\hline 4.1 - Responsabilidade sócio-ambiental & 30 \\
\hline 4.2 - Ética e desenvolvimento social & 30 \\
\hline 5 - Informações e Conhecimento & 60 \\
\hline 5.1 - Gestão das informações da organização & 20 \\
\hline 5.2 - Gestão das informações comparativas & 20 \\
\hline 5.3 - Gestão do capital intelectual & 20 \\
\hline 6 - Pessoas & 90 \\
\hline 6.1 - Sistemas de trabalho & 30 \\
\hline 6.2 - Capacitação e desenvolvimento & 30 \\
\hline 6.3 - Qualidade de vida & 30 \\
\hline 7 - Processos & 90 \\
\hline $\begin{array}{l}\text { 7.1 - Gestão de processos relativos aos pro- } \\
\text { dutos }\end{array}$ & 30 \\
\hline 7.2 - Gestão de processos de apoio & 20 \\
\hline $\begin{array}{l}7.3 \text { - Gestão de processos relativos aos forne- } \\
\text { cedores }\end{array}$ & 20 \\
\hline 7.4 - Gestão econômico-financeira & 20 \\
\hline 8 - Resultados & 450 \\
\hline $\begin{array}{l}8.1 \text { - Resultados relativos aos clientes e ao } \\
\text { mercado }\end{array}$ & 100 \\
\hline 8.2 - Resultados econômico-financeiros & 100 \\
\hline 8.3 - Resultados relativos às pessoas & 60 \\
\hline 8.4 - Resultados relativos aos fornecedores & 30 \\
\hline $\begin{array}{l}8.5 \text { - Resultados dos processos relativos aos } \\
\text { produtos }\end{array}$ & 80 \\
\hline 8.6 - Resultados relativos à sociedade & 30 \\
\hline $\begin{array}{l}8.7 \text { - Resultados dos processos de apoio e } \\
\text { organizacionais }\end{array}$ & 50 \\
\hline total & 1.000 \\
\hline
\end{tabular}

e quantidades, produzindo-se apenas contra pedidos firmes e não contra produção; $\mathrm{e}$

- Busca da perfeição. Retorna-se ao primeiro passo e se inicia a próxima transformação enxuta, criando um ciclo permanente e sustentado de melhoria. 
Silva e Ribeiro (2002), Lovelock e Wright (2001), Shah e Ward (2003) e Heskett (2002) reforçam a importância do entendimento do valor que um produto pode agregar ao cliente e à organização. Sobre este aspecto, Schonberger (1990) descreve a importância da empresa ter seu foco voltado, primeiramente, para o cliente e, em segundo lugar, para o produto. $\mathrm{O}$ foco no cliente deve ser prioridade, pois isso irá gerar oportunidades de melhorias nos processos, mas com o objetivo de solucionar problemas de todas as ordens que possam vir a destruir a relação cliente-fornecedor. Foco no cliente implica necessariamente conhecer sua percepção de valor. A efetiva implementação dos princípios relativos à mentalidade enxuta necessita apoio e suporte da alta direção da organização e estrutura organizacional.

Borchardt (2005) faz uma revisão compreensiva da mentalidade enxuta e propõe um método de geração de diretrizes para sua aplicação na indústria do transporte coletivo.

\section{A pesquisa: uma estrutura flexível para avaliação de desempenho estratégico}

O objetivo geral de pesquisa é propor uma metodologia para construir uma estrutura de avaliação de desempenho estratégico que seja exaustiva e flexível, de modo a conter todos os aspectos de uma estratégia e que seja modificável sempre que o ambiente exigir. Em uma perspectiva sistêmica, esta estrutura seria o elo de realimentação da execução da estratégia.

Escolheu-se a mentalidade enxuta como a fundamentação teórica a ser objeto de teste, pois, até quanto se investigou, não se achou uma estrutura de medições capaz de realimentar a inserção de aspectos da teoria da mentalidade enxuta a uma estratégia existente. Como há uma teoria, pareceu ser possível chegar a uma estrutura arborescente capaz de descrever os aspectos desta teoria a serem aplicados a um objeto. Para este objeto, escolheu-se a indústria do transporte coletivo de uma capital regional brasileira, pois as suas empresas têm uma homogeneidade de propósitos e muitas delas têm adotado modelos de avaliação de desempenho similares e comparáveis.

Antes de se avançar, deseja-se conectar o objetivo atual, a avaliação da execução estratégica, com o próximo passo da pesquisa, a formulação de uma estratégia de operação em transporte coletivo, cujas decisões foram tomadas com base na teoria da mentalidade enxuta.

Para Bonney (2000), uma estratégia de produção deve satisfazer as necessidades de clientes e da empresa e respeitar restrições típicas de mercado, tais como restrições legais, ambientais, técnicas e financeiras. Paiva et al. (2004) apontam categorias de decisões que devem ser tomadas na formulação da estratégia de produção ou opera- ções. As respostas às questões de decisão devem ser dadas segundo uma análise ambiental e segundo as crenças dos estrategistas. A formulação da estratégia responde questões que, ao serem formuladas, recompõem o ambiente de negócios e as crenças dos estrategistas. A inserção de aspectos enxutos em uma estratégia de operação existente é, portanto, composta pela formulação de questões que recomponham e integrem a teoria ao cenário objetivado e pela busca de respostas a estas questões. A atual parte da pesquisa formula as questões que recompõem e adaptam a teoria da mentalidade enxuta à indústria do transporte coletivo, sob a forma de uma estrutura arborescente ponderada. A resposta às questões surge na continuidade das pesquisas.

A metodologia de trabalho empregada na parte atual da pesquisa foi:

- Fazer uma pesquisa exploratória inicial acerca da situação da indústria escolhida;

- A partir da teoria da mentalidade enxuta, montar uma estrutura arborescente capaz de representar os condicionantes de tal sistema de gestão;

- Identificar especialistas capazes de, por meio de julgamentos, ponderar a estrutura para três empresas da indústria e para a indústria como um todo;

- Testar a estrutura em duas empresas, comparando-a com uma estrutura existente; $\mathrm{e}$

- Concluir compreensivamente a respeito dos resultados e do processo.

\subsection{Situação da indústria}

A indústria do transporte coletivo joga importante papel na sociedade brasileira. Segundo a ANTP (2003), mais de vinte e cinco milhões de brasileiros dependem do transporte coletivo por ônibus, o que indica a dimensão social desta indústria no Brasil. Devido a fatores estruturais que desestimulam o transporte coletivo, a indústria enfrenta uma dura perda de viagens remuneradas, que caíram de 433 milhões em 1994 para apenas 295 milhões em 2001. Esta queda compromete a rentabilidade do setor e aponta para um acréscimo nas tarifas e redução na prestação do serviço, com graves consequiências sociais para seus usuários.

Esta redução nas viagens ocorre em quase todas as capitais brasileiras, sendo temporariamente revertida em duas delas, após transformações estruturais importantes. Estes casos de reversão se devem a iniciativas, tais como a transferência não-onerosa entre linhas e a bilhetagem automática. Absorvidas estas transformações, a tendência de queda retornou. Verifica-se a necessidade de mudanças gerenciais nas empresas, de modo a torná-las potencialmente capazes de sobreviver à redução de faturamento e, na seqüência, retomar o crescimento. 
Diversas alternativas têm sido tentadas no setor, tais como os modelos gerenciais do PNQ, do PGQP e da ANTP, todos baseados no prêmio Malcolm Baldridge, ou a certificação ISO 9000. Estas alternativas incorporam aspectos de qualidade de conformação à estratégia de operação existente. Outra alternativa é a incorporação à atual estratégia de aspectos de redução de custos. Entende-se que a teoria da mentalidade enxuta possa fundamentar estes aspectos. Como o objeto que cria valor na indústria não é tangível, como na fabricação, a teoria deve ser adaptada à realidade do setor para que possa gerar um modelo de gestão aplicável.

Estudos qualitativos e ações de consultoria preliminares tornaram claro que o entendimento e a aceitação de uma estratégia de produção enxuta não seria simples na indústria. Antes de se propor um modelo útil de gestão, os princípios da produção enxuta deveriam ser, de algum modo, adaptados à particular realidade em que seriam aplicados. Os pesquisadores concluíram que era necessário determinar a capacidade da indústria em entender os princípios da produção enxuta, avaliando seu grau de preparação para receber um futuro modelo específico de gestão. Esta parte foi concluída e apresentada em Sellitto et al. (2003). O segundo passo é a construção de um sistema de avaliação do desempenho estratégico, ora apresentado, e, ao fim, vem a formulação das modificações na estratégia de operação.

A indústria estudada se sedia em uma capital brasileira e é composta por quinze empresas, mais a associação de classe e o órgão gestor público de regulação, que mantém entre si relações complexas e imbricadas, condicionantes da estratégia de operação. As empresas formam consórcios geográficos, que previnem a competição destrutiva e podem cooperar em aspectos logísticos e de manutenção, mas esta cooperação ainda não atingiu um nível satisfatório, devido a fatores que não serão investigados nesta pesquisa.

\subsection{Montagem da estrutura arborescente ponderada}

Para estruturar a inserção dos aspectos enxutos na estratégia atual, usaram-se os cinco princípios como construtos, assumindo-se que tenham mútua independência, como no PNQ. Ou seja, o desempenho em um nível inferior afeta única e exclusivamente o nível superior, sem não-linearidades, sombreamentos ou circularidades. Estas assunções não correspondem exatamente à realidade, pois há, na realidade complexa, mútuas interferências. Reconhecem-se e admitem-se tais simplificações.

O primeiro nível da estrutura arborescente é constituído do termo de topo Mentalidade Enxuta, suportado pelos cinco princípios. Para o segundo nível, foram construídos, em conjunto com especialistas da indústria, mapas cognitivos, segundo a técnica apresentada em Ensslin et al. (2001) e que não será revisada. Foram identificados seis pontos de vista fundamentais para cada ente. Esta parte da pesquisa é apresentada em Sellitto et al. (2003). A estrutura completa é apresentada no Quadro 1.

A seguir passou-se à ponderação da estrutura. Oito especialistas construíram o primeiro nível, sendo quatro especialistas em mentalidade enxuta, com experiência em transporte, e quatro especialistas em transporte, com experiência em mentalidade enxuta. Todos possuem especialização, mestrado ou doutorado compatíveis com a indústria.

O segundo nível foi construído por doze especialistas, todos com formação superior balanceada entre ciências humanas e ciências exatas e experiências profissionais balanceadas entre empresas operadoras, órgãos reguladores e empresariado, segundo a Tabela 2. Dois dos respondentes são executivo e diretor da empresa $\mathrm{A}$, três são executivos e diretor da empresa $\mathrm{B}$ e um é, ao mesmo tempo, executivo da empresa $\mathrm{C}$ e empresário de prestação de serviços na indústria. Os demais respondentes são especialistas em regulação dos serviços e em prestação de serviços na indústria, sem ligação exclusiva com uma empresa.

As respostas de primeiro nível geram a ponderação e as razões de consistência $C R$ da Tabela 3. A $C R$ é a probabilidade de que a distribuição alcançada seja aleatória, não se originando de julgamento de decisor. Os coeficientes de variação $c v=\sigma / \mu$ são baixos. Chega-se à expressão linear da Equação 1, representante da média do pensamento dos decisores, e que se constitui em uma função-objetivo para a implantação de uma estratégia de operação baseada na teoria da mentalidade enxuta na indústria estudada.

produção enxuta $=0,467$.

[especificação de valor] $+0,136$.[fluxo contínuo] +

0,170.[fluxo balanceado] $+0,080$. [produção puxada $]+$ 0,147.[melhoria contínua]

As respostas de segundo nível geraram as Tabelas 4 e 5. Os respondentes usaram as inconsistências de suas respostas na reformulação de seus julgamentos.

Na Tabela 4, são apresentadas as médias, os desvios-padrão e os coeficientes de variação dos pesos ponderados. A soma dos pesos dos seis conceitos de um construto totaliza 1. As médias obtidas são uma distribuição de importâncias relativas de conceitos dentro do construto. Na Tabela 5, estão as respostas dos respondentes das empresas A, $\mathrm{B}$ e $\mathrm{C}$ individualmente e as respostas gerais da indústria. Os valores foram obtidos multiplicando-se a importância relativa de cada conceito dentro do construto de primeiro nível pela importância relativa do construto. A soma dos valores de cada conceito iguala o valor do respectivo construto. Os valores atribuídos aos conceitos constituem uma 
Quadro 1. Estrutura representativa de uma estratégia de operação enxuta em transporte coletivo.

\begin{tabular}{|c|c|c|}
\hline Termo de topo & Primeiro nível & Segundo nível \\
\hline \multirow{30}{*}{$\begin{array}{l}\text { Mentalidade } \\
\text { enxuta }\end{array}$} & \multirow{6}{*}{$\begin{array}{l}\text { Análise de valor: } \\
\text { capacidade de compreender o } \\
\text { que agrega valor ao cliente ou usuário do } \\
\text { serviço }\end{array}$} & Conhecimento das características dos clientes \\
\hline & & Conhecimento das finalidades dos clientes \\
\hline & & Conhecimento dos fatores de decisão dos clientes \\
\hline & & Conhecimento dos momentos de tomada de serviços \\
\hline & & Conhecimento dos ritmos de tomada de serviços \\
\hline & & Conhecimento dos locais de tomada de serviços \\
\hline & \multirow{6}{*}{$\begin{array}{l}\text { Fluxo contínuo: } \\
\text { capacidade de eliminar perdas na cadeia de } \\
\text { produção de valor da indústria }\end{array}$} & Conhecimento do conceito de perda \\
\hline & & Capacidade de identificar suas perdas \\
\hline & & Capacidade de tipificar suas perdas \\
\hline & & Capacidade de medir suas perdas \\
\hline & & Capacidade de eliminar suas perdas \\
\hline & & Capacidade de entender a importância da velocidade \\
\hline & \multirow{6}{*}{$\begin{array}{l}\text { Fluxo balanceado: } \\
\text { capacidade de tornar a operação previsível }\end{array}$} & Conhecimento de seus processos \\
\hline & & Importância dada ao mapeamento de seus processos \\
\hline & & Importância dada à medição de processos internos \\
\hline & & Importância dada à medição de processos de parceiros \\
\hline & & Vontade em mudar processos \\
\hline & & Capacidade de entender a multifuncionalidade \\
\hline & \multirow{6}{*}{$\begin{array}{l}\text { Produção puxada: } \\
\text { capacidade de permitir que o cliente "puxe" } \\
\text { a operação }\end{array}$} & Capacidade de ouvir a voz do cliente \\
\hline & & Consideração dada à voz do cliente \\
\hline & & Importância dada à voz do cliente \\
\hline & & Capacidade de aproveitar a voz do cliente \\
\hline & & Vontade de aproveitar a voz do cliente \\
\hline & & Intensidade da interação com o cliente \\
\hline & \multirow{6}{*}{$\begin{array}{l}\text { Melhoria contínua: } \\
\text { capacidade de medir e melhorar o resultado } \\
\text { continuamente }\end{array}$} & Conhecimento do conceito de melhoria contínua \\
\hline & & Capacidade de promover a melhoria contínua \\
\hline & & Capacidade de focar a melhoria contínua \\
\hline & & Capacidade de controlar a melhoria contínua \\
\hline & & Importância dada à melhoria contínua \\
\hline & & Intensidade da melhoria contínua \\
\hline
\end{tabular}

Tabela 2. Distribuição balanceada dos respondentes do segundo nível.

\begin{tabular}{cccc}
\hline Formação e & \multicolumn{3}{c}{ Atividade principal } \\
interesse & Empresário & Operador & Regulador \\
\hline Mais técnica & 2 & 2 & 2 \\
Mais humanística & 2 & 2 & 2 \\
\hline
\end{tabular}

distribuição da importância relativa atribuída ao construto de origem entre os conceitos a este subordinados.

Por exemplo, o primeiro construto da estratégia de operação enxuta, a especificação de valor, tem uma importância relativa de $47,6 \%$, determinada na primeira parte do estudo. Este valor é distribuído de quatro modos diferentes, segundo a preferência dos decisores. Os decisores da empresa A distribuíram esta importância segundo a terceira coluna da Tabela 5, o mesmo valendo para as empresas B e C. Na última coluna desta tabela, está a distribuição atribuída pela média dos doze especialistas e que pode ser considerada como a distribuição média, para a indústria, da importância relativa deste construto entre seus conceitos.

\section{Discussão}

As opiniões dos oito especialistas que construíram o primeiro nível apresentam coeficientes de variação médio $(c v m)$ de 0,45 . As opiniões dos doze especialistas que construíram o segundo nível apresentam $c v m$ de 0,79 . As opiniões dos dois decisores da empresa A apresentam um $c v m$ de 0,47 , enquanto que, para os três decisores da empresa B, o cvm é de 0,65. Eliminando o decisor mais divergente de $\mathrm{B}$, o $c v m$ cai para 0,50. A empresa $\mathrm{C}$ foi avaliada por um único decisor, não havendo variação. $\mathrm{O}$ cvm dos doze decisores foi de 0,79 .

Estes valores sugerem que o primeiro nível da estrutura de medição seja mais robusto, o que já era esperado, pois são menos decisores e menos critérios de decisão. A análise sugere que o primeiro nível seja mantido para posteriores modelos. Já no segundo nível, também como 
Tabela 3. Coeficientes do primeiro nível da estrutura de medição.

\begin{tabular}{|c|c|c|c|c|c|c|}
\hline & $\begin{array}{l}\text { Especificação } \\
\text { de valor }\end{array}$ & $\begin{array}{c}\text { Fluxo } \\
\text { contínuo }\end{array}$ & $\begin{array}{c}\text { Fluxo } \\
\text { balanceado }\end{array}$ & $\begin{array}{l}\text { Produção } \\
\text { puxada }\end{array}$ & $\begin{array}{l}\text { Melhoria } \\
\text { contínua }\end{array}$ & $C R$ \\
\hline \multirow{8}{*}{$\begin{array}{l}\text { Coeficientes calculados a partir das } \\
\text { preferências de oito especialistas }\end{array}$} & 0,49 & 0,20 & 0,10 & 0,11 & 0,10 & 0,09 \\
\hline & 0,49 & 0,22 & 0,05 & 0,14 & 0,10 & 0,11 \\
\hline & 0,44 & 0,08 & 0,27 & 0,06 & 0,15 & 0,09 \\
\hline & 0,46 & 0,17 & 0,17 & 0,17 & 0,04 & 0,01 \\
\hline & 0,36 & 0,09 & 0,34 & 0,04 & 0,17 & 0,08 \\
\hline & 0,48 & 0,18 & 0,18 & 0,06 & 0,11 & 0,05 \\
\hline & 0,51 & 0,06 & 0,13 & 0,03 & 0,26 & 0,07 \\
\hline & 0,49 & 0,09 & 0,13 & 0,04 & 0,25 & 0,07 \\
\hline Média & 0,467 & 0,136 & 0,170 & 0,080 & 0,147 & 0,07 \\
\hline Coeficiente de variação & 0,11 & 0,45 & 0,55 & 0,63 & 0,53 & 0,43 \\
\hline
\end{tabular}

Tabela 4. Distribuição de importância relativa dos conceitos dentro dos construtos.

\begin{tabular}{|c|c|c|c|c|}
\hline Primeiro nível & Segundo nível & Média & Desvio-padrão & cv \\
\hline \multirow[t]{6}{*}{ Análise de valor } & Conhecimento das características dos clientes & 0,21 & 0,18 & 0,86 \\
\hline & Conhecimento das finalidades dos clientes & 0,15 & 0,15 & 1,00 \\
\hline & Conhecimento dos fatores de decisão dos clientes & 0,21 & 0,18 & 0,83 \\
\hline & Conhecimento dos momentos de tomada de serviços & 0,17 & 0,09 & 0,56 \\
\hline & Conhecimento dos ritmos de tomada de serviços & 0,15 & 0,14 & 0,94 \\
\hline & Conhecimento dos locais de tomada de serviços & 0,11 & 0,08 & 0,74 \\
\hline \multirow[t]{6}{*}{ Fluxo contínuo } & Conhecimento do conceito de perda & 0,23 & 0,19 & 0,82 \\
\hline & Capacidade de identificar suas perdas & 0,20 & 0,13 & 0,65 \\
\hline & Capacidade de tipificar suas perdas & 0,13 & 0,10 & 0,78 \\
\hline & Capacidade de medir suas perdas & 0,10 & 0,04 & 0,41 \\
\hline & Capacidade de eliminar suas perdas & 0,18 & 0,15 & 0,87 \\
\hline & Capacidade de entender a importância da velocidade & 0,17 & 0,15 & 0,89 \\
\hline \multirow[t]{6}{*}{ Fluxo balanceado } & Conhecimento de seus processos & 0,29 & 0,19 & 0,66 \\
\hline & Importância dada ao mapeamento de seus processos & 0,17 & 0,11 & 0,64 \\
\hline & Importância dada à medição de processos internos & 0,13 & 0,08 & 0,65 \\
\hline & Importância dada à medição de processos de parceiros & 0,13 & 0,14 & 1,07 \\
\hline & Vontade em mudar processos & 0,12 & 0,11 & 0,92 \\
\hline & Capacidade de entender a multifuncionalidade & 0,16 & 0,14 & 0,85 \\
\hline \multirow[t]{6}{*}{ Produção puxada } & Capacidade de ouvir a voz do cliente & 0,18 & 0,16 & 0,89 \\
\hline & Consideração dada à voz do cliente & 0,13 & 0,07 & 0,56 \\
\hline & Importância dada à voz do cliente & 0,23 & 0,17 & 0,77 \\
\hline & Capacidade de aproveitar a voz do cliente & 0,16 & 0,12 & 0,76 \\
\hline & Vontade de aproveitar a voz do cliente & 0,17 & 0,13 & 0,8 \\
\hline & Intensidade da interação com o cliente & 0,14 & 0,14 & 1,03 \\
\hline \multirow[t]{6}{*}{ Melhoria contínua } & Conhecimento do conceito de melhoria contínua & 0,24 & 0,20 & 0,82 \\
\hline & Capacidade de promover a melhoria contínua & 0,21 & 0,13 & 0,62 \\
\hline & Capacidade de focar a melhoria contínua & 0,15 & 0,11 & 0,75 \\
\hline & Capacidade de controlar a melhoria contínua & 0,10 & 0,07 & 0,69 \\
\hline & Importância dada à melhoria contínua & 0,16 & 0,14 & 0,83 \\
\hline & Intensidade da melhoria contínua & 0,13 & 0,13 & 1,01 \\
\hline
\end{tabular}

era esperado, quanto mais genérico o modelo desejado mais variação as opiniões apresentam.

Na Figura 1, apresenta-se um dendograma de similaridade para os doze decisores, obtido com o apoio do software SPSS v. 10.0.1. As variáveis são os coeficientes atribuídos aos conceitos pelos decisores. Os decisores das empresas A e B mantêm mais similaridade entre si do que com os demais decisores. $\mathrm{O}$ decisor de $\mathrm{C}$ se distancia dos 
Tabela 5. Estruturas de medição para as três empresas e para a indústria.

\begin{tabular}{|c|c|c|c|c|c|}
\hline Construtos e conceitos & $\begin{array}{c}\text { Peso do } \\
\text { construto }\end{array}$ & $\begin{array}{c}\text { Empresa } \\
\mathbf{A} \\
\end{array}$ & $\begin{array}{c}\text { Empresa } \\
\text { B } \\
\end{array}$ & $\begin{array}{c}\text { Empresa } \\
\mathrm{C} \\
\end{array}$ & $\begin{array}{l}\text { Geral da } \\
\text { indústria }\end{array}$ \\
\hline \multicolumn{6}{|l|}{ Análise de valor } \\
\hline Conhecimento das características dos clientes & 467 & 30 & 68 & 193 & 99 \\
\hline Conhecimento das finalidades dos clientes & & 99 & 102 & 80 & 71 \\
\hline Conhecimento dos fatores de decisão dos clientes & & 57 & 144 & 116 & 99 \\
\hline Conhecimento dos momentos de tomada de serviços & & 143 & 99 & 43 & 77 \\
\hline Conhecimento dos ritmos de tomada de serviços & & 89 & 30 & 22 & 69 \\
\hline Conhecimento dos locais de tomada de serviços & & 49 & 24 & 13 & 52 \\
\hline \multicolumn{6}{|l|}{ Fluxo contínuo } \\
\hline Conhecimento do conceito de perda & 136 & 17 & 19 & 62 & 31 \\
\hline Capacidade de identificar suas perdas & & 35 & 39 & 29 & 27 \\
\hline Capacidade de tipificar suas perdas & & 25 & 9 & 8 & 17 \\
\hline Capacidade de medir suas perdas & & 14 & 15 & 19 & 14 \\
\hline Capacidade de eliminar suas perdas & & 33 & 40 & 12 & 24 \\
\hline Capacidade de entender a importância da velocidade & & 12 & 14 & 6 & 23 \\
\hline \multicolumn{6}{|l|}{ Fluxo balanceado } \\
\hline Conhecimento de seus processos & 170 & 67 & 40 & 77 & 48 \\
\hline Importância dada ao mapeamento de seus processos & & 14 & 33 & 37 & 29 \\
\hline Importância dada à medição de processos internos & & 26 & 28 & 7 & 22 \\
\hline Importância dada à medição de processos de parceiros & & 12 & 26 & 26 & 22 \\
\hline Vontade em mudar processos & & 26 & 15 & 12 & 21 \\
\hline Capacidade de entender a multifuncionalidade & & 25 & 28 & 11 & 28 \\
\hline \multicolumn{6}{|l|}{ Produção puxada } \\
\hline Capacidade de ouvir a voz do cliente & 80 & 14 & 3 & 34 & 15 \\
\hline Consideração dada à voz do cliente & & 6 & 4 & 11 & 10 \\
\hline Importância dada à voz do cliente & & 34 & 27 & 3 & 18 \\
\hline Capacidade de aproveitar a voz do cliente & & 9 & 13 & 18 & 13 \\
\hline Vontade de aproveitar a voz do cliente & & 13 & 19 & 9 & 13 \\
\hline Intensidade da interação com o cliente & & 4 & 14 & 5 & 11 \\
\hline \multicolumn{6}{|l|}{ Melhoria contínua } \\
\hline Conhecimento do conceito de melhoria contínua & 147 & 30 & 8 & 67 & 35 \\
\hline Capacidade de promover a melhoria contínua & & 30 & 44 & 30 & 32 \\
\hline Capacidade de focar a melhoria contínua & & 36 & 28 & 7 & 22 \\
\hline Capacidade de controlar a melhoria contínua & & 9 & 7 & 21 & 15 \\
\hline Importância dada à melhoria contínua & & 34 & 37 & 11 & 24 \\
\hline Intensidade da melhoria contínua & & 8 & 23 & 11 & 19 \\
\hline Total geral & 1.000 & 1.000 & 1.000 & 1.000 & 1.000 \\
\hline
\end{tabular}

decisores de A e B. Um corte em $65 \%$ aglomera os cinco decisores de A, B e C.

As empresas da indústria adotam um procedimento estratégico semelhante, de certa forma estimulado pela organização de classe. Pela análise do cenário, formulam objetivos, estratégias e planos, estabelecendo um referencial de avaliação de desempenho da estratégia. A indústria é bem-sucedida, alguns de seus membros participam com destaque do prêmio setorial da ANTP e muitas em- presas adotam modelos de gestão baseados nos critérios de excelência do prêmio Malcolm Baldridge, como referencial de desempenho.

Uma análise que pode ser feita é a comparação entre a avaliação de desempenho da atual estratégia pelo método existente com o método ora proposto. A comparação será feita nas empresas B e C. As empresas auto-avaliaram-se por questionário, segundo uma escala de Likert, que investiga a impressão dos decisores sobre a situação 
dos trinta conceitos ora propostos [péssimo; ruim; médio; bom; ótimo], aos quais associaram-se valores de 1 a 5 . A auto-avaliação é apresentada e analisada em Sellitto, Borchardt e Pereira (2003).

Na Tabela 6, comparam-se os resultados das duas auto-avaliações por um teste estatístico. As respostas às trinta variáveis ora propostas são comparadas com a média das respostas ao modelo existente. A hipótese nula $H_{0}=[(E E-M B)=0]$ propõe que a troca do sistema de avaliação não afete o resultado. $E E$ é o resultado da autoavaliação ora proposta, a estratégia enxuta, com trinta parcelas, e $M B$ é o resultado normalizado da auto-avaliação vigente, calculado pela média das atuais variáveis categóricas. Um eventual viés é compensado, pois se tratam dos mesmos avaliadores. Com um nível de confiança de $95 \%$, afirma-se que, em ambas as empresas, a troca de sistema de avaliação muda o resultado da avaliação do desempenho estratégico.

\section{Conclusões}

O objetivo deste trabalho foi a construção de uma estrutura de avaliação de desempenho estratégico que fosse, ao mesmo tempo, capaz de realimentar ações estratégicas e flexível o suficiente para ser modificada sempre que o ambiente estratégico exigir. A flexibilidade é conferida pelo procedimento metodológico adotado, que pode ser replicado sempre que a teoria subjacente ou o ambiente estratégico mudar. $\mathrm{O}$ procedimento proposto foi testado na construção de uma estrutura para avaliar a introdução de aspectos enxutos em uma estratégia de operação existente na indústria de transporte coletivo.

Desenvolveu-se uma metodologia para a modelagem das crenças de estrategistas bem sucedidos na indústria em estudo e conhecedores da teoria da mentalidade enxuta. Chegou-se a uma estrutura hierárquica de avaliação de desempenho geral para a indústria e estruturas específicas para as três empresas estudadas. Duas das empresas usam como realimentador de ações estratégicas um sistema de avaliação baseado nos critérios do prêmio Malcolm Baldridge. Em ambas as empresas, a troca do sistema produziu uma mudança com nível de 5\% de significância no resultado da avaliação, o que indica a especificidade da estrutura proposta.

Conclui-se que, no caso, dois sistemas de avaliação diferentes conduziram a resultados diferentes, o que pode gerar planos de ações diferentes para os mesmos objetivos estratégicos. Outra conclusão é que, quando o objeto foi uma única empresa, a variação entre as opiniões foi menor do que quando o objeto incluiu três empresas. Deste modo abre-se uma frente de pesquisa: até que ponto modelos invariantes e apriorísticos descrevem interesses de empresas sujeitas a diferentes condicionantes competitivos? Uma decorrência deste questionamento é a possibi-

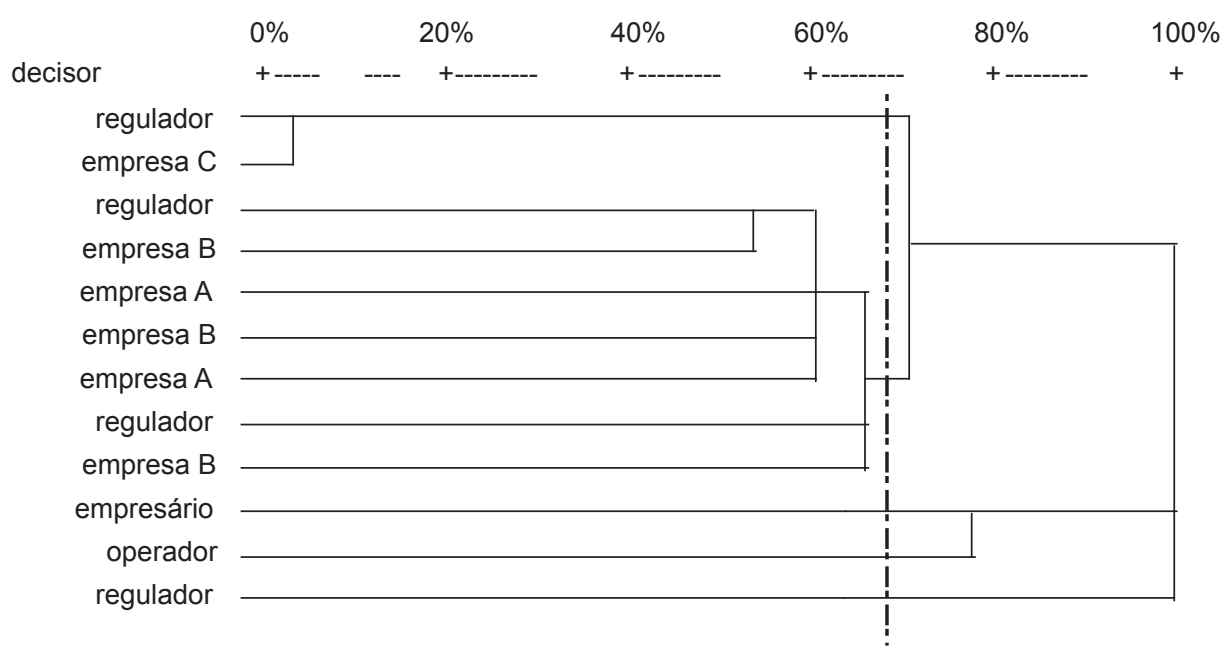

Figura 1. Dendograma de similaridade de decisores.

Tabela 6. Comparação entre as auto-avaliações com base em modelos distintos.

\begin{tabular}{cccc}
\hline Empresa & $\begin{array}{c}\text { Modelo do prêmio Malcolm } \\
\text { Baldridge, em uso }\end{array}$ & $\begin{array}{c}\text { Modelo da estratégia de } \\
\text { operação enxuta, proposto }\end{array}$ & $\begin{array}{c}\text { Teste } t \text { para } \alpha=\mathbf{5 \%} \text { e } v=\mathbf{2 9} \\
t_{\text {crítico }}=\mathbf{2 , 3 6}\end{array}$ \\
\hline $\mathrm{B}$ & $95 \%$ & $87,1 \%$ & $t=-2,93 ;$ valor $-p=1 \%$; difere \\
$\mathrm{C}$ & $88 \%$ & $73,9 \%$ & $t=-4,42 ;$ valor $-p=0 \%$; difere \\
\hline
\end{tabular}


lidade de uso de mapas cognitivos e métodos multicriteriais de apoio à decisão na modificação e manutenção de modelos fortemente estruturados, tais como o PNQ.

A presente pesquisa é a segunda de três etapas de um projeto de pesquisa em operações industriais.

Na primeira etapa, montou-se e testou-se uma estrutura arborescente capaz de representar aspectos enxutos em uma estratégia de operação de empresas de transporte coletivo.

$\mathrm{Na}$ atual etapa, calcularam-se os coeficientes da estrutura, chegando-se a um sistema de avaliação de resultado destes aspectos para a indústria e para três empresas em particular. Destaca-se que o uso do AHP exige cuidados quanto à inconsistência de resultados, devendo-se adotar uma inconsistência menor do que $10 \%$, o que pode se conseguir flexibilizando a escala original de valores sugerida por Saaty (1991). Cuidados também devem ser tomados se critérios forem inseridos ou retirados da estrutura. Todos os julgamentos devem ser repetidos e o problema toma outro formato, totalmente diferente. No entanto, reforça-se que a parte metodológica qualitativa permite a formação de uma estrutura padronizada capaz de apreender aspectos de uma complexidade. Outros métodos multicriteriais podem ser testados na continuidade ou eventualmente acoplados ao AHP, como o fizeram Liu e Hai (2005).

A última etapa formulará os planos para a introdução dos aspectos enxutos na estratégia de operação da indústria. Encerrada a pesquisa, ter-se-ão elementos suficientes para a proposição de um modelo de gestão que insira a mentalidade enxuta na operação do transporte coletivo.

\section{Reconhecimentos}

Os autores reconhecem a importância do papel dos participantes da pesquisa: Associação dos Transportadores de Passageiros de Porto Alegre, empresas de transporte coletivo de Porto Alegre, EPTC, Cia Carris PortoAlegrense, Exata Logística e consultores autônomos de competência reconhecida nacional e internacionalmente. Deseja-se, também, reconhecer a importância da Universidade do Vale do Rio dos Sinos pelo financiamento da pesquisa. Reconhece-se, por fim, a relevância das recomendações dos pareceristas anônimos, que muito contribuíram para o estado final do artigo.

\section{Referências Bibliográficas}

ANTP - Associação Nacional de Transportes Públicos. O transporte na cidade do século 21. S. Paulo: 2001. Disponível http://www.antp.org.br/TELAS/transporte/transporte.htm. Acesso em: 20 Abr. 2003.

ARANTES, N. Sistemas de gestão empresarial: conceitos permanentes na administração de empresas válidas. S. Paulo: Atlas, 1998.

BITITCI, U. Modelling of performance measurement syste$\mathrm{ms}$ in manufacturing enterprises, International Journal of Production Economics, v. 42, n. 2, p. 137-147, 1995.

BITITCI, U.; SUWIGNJO, P.; CARRIE, A. Strategy management through quantitative modelling of performance measurement systems. International Journal of Production Economics, v. 69, n. 1, p. 15-22, 2001.

BITITCI, U.; NUDURUPATI, S.; TURNER, T.; CREIGHTON, S. Web enable performance measurements: management implications. International Journal of Operations and Production Management, v. 22, n. 11, p. 1273-1287, 2002.

BNQP: Baldrige National Quality Program. Criteria for performance excelence. Disponível http://www.quality.nist.gov/Business_Criteria.htm. Acesso em: 14 Nov. 2003.

BONNEY, M. Control of manufacturing systems. Produto \& Produção, v. 4, n. 1, p. 1-16, 2000.
BORCHARDT, M. Diretrizes para a implementação dos princípios da Mentalidade Enxuta: o caso das empresas de transporte coletivo rodoviário urbano. Tese (doutorado em Engenharia de Produção). Programa de Pós-graduação em Engenharia de Produção, UFSC, Florianópolis. 2005.

BOURNE, M.; MILLS, J.; WILCOX, M.; NEELY, A.; PLATTS, K. Designing, implementing and updating performance measurements systems. International Journal of Operations and Production Management, v. 20, n. 7, p. 754-771, 2000.

DYER, J. Remarks on the Analytic Hierarchy Process. Management Science, v. 36, n. 3, p. 249-258, 1990a.

DYER, J. A clarification of "Remarks on the Analytic Hierarchy Process". Management Science, v. 36, n. 3, p. $274-275,1990$ b.

EVANS, J. An exploratory study of performance measurement systems and relationships with performance results. Journal of Operations Management, v. 22, n. 3, p. 219-232, 2004.

ENSSLIN, L.; MONTIBELLER, G.; NORONHA, S. Apoio à decisão. Florianópolis: Insular, 2001.

FPNQ: FUNDAÇÃO DO PRÊMIO NACIONAL DA QUALIDADE. Critérios de Excelência 2004. Disponível em: <http//www.fpnq.org.br/>. Acesso em: 1 Mai. 2004. 
FRENCH, S. Decision Theory: an introduction to the mathematics of rationality, Ed. Ellis Horwood, Chichester, West Sussex, UK, 1986.

GASPARETTO, V. Proposta de uma sistemática para avaliação de desempenho em cadeias de suprimentos. Tese de doutorado. EPS-UFSC, Florianópolis. 2003.

GOMES, C.; YASIM, M.; LISBOA, J. An examination of manufacturing organizations's performance evaluation: analysis, implications and a framework for future research. International Journal of Operations and Production Management, v. 24, n. 5, p. 488-513, 2004.

GOMES, L; ARAYA, M.; CARIGNANO, C. Tomada de decisão em cenários complexos. S. Paulo: Thomson Learning, 2004.

HANSEN, P. Um modelo meso-analítico de medição de desempenho competitivo de cadeias produtivas. Tese (Doutorado em Engenharia de Produção) - Escola de Engenharia, UFRGS, P. Alegre, 2004.

HARKER, P.; VARGAS, L. Reply "Remarks on the Analytic Hierarchy Process" by J. S. Dyer. Management Science, v. 36, n. 3, p. 269-273, 1990.

HESKETT, J. Lucro na prestação de serviços: como crescer com a lealdade e a satisfação dos clientes. R. Janeiro: Campus, 2002.

HRONEC, S. Vital Signs - Using quality, time, and cost performance measurements to chart your company's future. USA: Arthur Andersen Co, 1993.

KAPLAN, R.; NORTON, D. A estratégia em ação. R. Janeiro: Campus, 1997.

KASUL, R.; MOTWANI, J. Performance measurements in world-class operations: a strategic model. Benchmarking for Quality Management and Technology, v. 2, n. 2, p. 20-36, 1995.

KENNERLY, M.; NEELY, A. Measuring performance in a changing business environment. International Journal of Operations and Production Management, v. 23, n. 2, p. 213-229, 2003.

KEENEY, R.; RAIFFA, H.: Decisions with multiples objectives: preferences and value trade-offs. N. York: John Wiley and Sons, 1976.

LIMA, V. Regulador e Regulados: análise do processo de avaliação de desempenho das empresas do sistema municipal de transporte por ônibus de Belo Horizonte. Tese (Doutorado em Administração) - UFMG, B. Horizonte, 1999.

LIMA, E. Modelo multicriterial para análise e avaliação de sistemas de transportes coletivos com ônibus. Dissertação de mestrado em engenharia civil - Universidade Federal da Paraíba. Campina Grande. 2000.
LIU, F.; HAI, H. The voting analytic hierarchy process method for selecting supplier. International Journal of Production Economics, v. 97, n. 3, p. 308-317, 2005.

LOHMAN, C.; FORTUIN, L.; WOUTERS, M. Designing a performance measurement system: a case study. European Journal of Operational Research, v. 156, n. 2, p. 267-286, 2004.

LOVELOCK, C.; WRIGHT, L. Serviços: marketing e gestão. S. Paulo: Saraiva, 2001.

MARTINS, R. Sistemas de medição de desempenho: um modelo para estruturação do uso. Tese de doutorado. Escola Politécnica. S. Paulo. USP. 1999.

MELLO, J.; GOMES, E.; LINS, M. Análise multicritério da presença da Universidade Federal Fluminense com o uso do método MACBETH. Produção, v. 11, n. 2, p. 53-67, 2002.

MELNICK, S.; STEWARD, D.; SWINK; M. Metrics and performance measurements in operations management: dealing with the metrics maze. Journal of Operations Management, v. 22, n. 3, p. 219-217, 2004.

MIRANDA, L.; SILVA, J. Medição de desempenho. In: SCHMIDT, P (Org.). Controladoria: agregando valor para a empresa. P. Alegre: Bookman, 2002.

MOREIRA, D. Dimensões do desempenho em manufatura e serviços. S. Paulo: Pioneira, 1996.

MÜLLER, C. Modelo de gestão integrando planejamento estratégico, sistema de avaliação de desempenho e gerenciamento de processo. Tese (doutorado em Engenharia de Produção) - PPGEP-UFRGS, P. Alegre. 2003.

PAIVA, E.; CARVALHO, L.; FENSTENSEIFER, J. Estratégia de produção e de operações. P. Alegre: Bookman, 2004.

PARTOVI, F.; WHITERS, B.; BRADFORD, J. How Tompkins rubber company used Analytic Hierarchy Process to enhance ISO-9000 related decision making, Production and Inventory Management Journal, v. 43, n. 1/2, p. 13-22, 2002.

SAATY, T. An exposition of the AHP in reply to the paper "Remarks on the Analytic Hierarchy Process". Management Science, v. 36, n. 3, p. 259-267, 1990.

SAATY, T. Método de análise hierárquica, S. Paulo: Makron Books do Brasil, 1991.

SALOMON, V. Auxílio à decisão para a adoção de políticas de compras, Produto \& Produção, v. 6, n. 1, p. 1-8, 2002.

SALOMON, V.; MONTEVECCHI, J. Método de análise em redes: sucessor do método de análise hierárquica? Produto \& Produção, v. 3, n. 2, p. 107-117, 1998. 
SCHONBERGER, R. Building a chain of customer. N. York: The Free Press, 1990.

SELLITTO, M. Medição e controle de desempenho estratégico em sistemas de manufatura. Tese (Doutorado em Engenharia de Produção) - Escola de Engenharia, UFRGS, P. Alegre, 2005.

SELLITTO, M.; BORCHARDT, M.; PEREIRA, G. Assessing the degree of promptness of a service industry to adopt lean thinking, REAd, v. 9, n. 6, p. 105-130, 2003.

SHAH, R.; WARD, P. Lean manufacturing: context, practice bundles and performance. Journal of Operations Management, v. 21, n. 2, p. 129-149, 2003.
SILVA, P.; RIBEIRO, J. Uma proposta para a modelagem do valor percebido na prestação de serviços. Produção, v. 12, n. 1, p. 18-33, 2002.

SUWIGNJO, P; BITITCI, U.; CARRIE, A. Quantitative models for performance measurements system. International Journal of Production Economics, v. 64, n. 1-3, p. 231-241, 2000.

TONCHIA, S. Linking performance measurement system to strategic and organizational choices. International Journal of Business Performance, v. 2, n. 1, p. 15-29, 2000.

WOMACK, J.; JONES, D. A mentalidade enxuta nas empresas, R. Janeiro: Campus, 1997.

\section{MULTICRITERIAL PERFORMANCE ASSESSMENT: A CASE STUDY IN THE URBAN PASSENGER TRANSPORTATION SECTOR}

Abstract

This paper proposes a multicriterial framework for strategic performance assessment, beginning with a presentation of concepts of performance measurement, and a description of management models based on the Malcolm Baldridge Quality Award and lean thinking. A method is then outlined for building a flexible framework for performance assessment and a case study is described: an evaluation of the performance of aspects of lean thinking in an urban passenger transportation operation. The paper concludes with a discussion of the findings of the case study using the proposed method, comparing it with the evaluation method currently used by two companies in the sector under study.

Keywords: multicriterial performance assessment, lean production performance assessment, urban passenger transportation performance assessment. 